\title{
ORIGINAL
}

\section{FACTORES ASOCIADOS CON EL USO Y ADECUACIÓN DE LA HOSPITALIZACIÓN EN PERSONAS MAYORES DE 64 AÑOS}

Francisco Suárez García (1), David Oterino de la Fuente (2), Salvador Peiró (2), Julián Librero (2), Carmen Barrero Raya (3), Nieves Parras García de León (3), M. ${ }^{a}$ Antonia Crespo Pérez (3) y Alejandro Peréz-Martín (3)

(1) Dirección General de Ordenación de Servicios Sanitarios. Consejería de Salud y Servicios Sanitarios del Principado de Asturias.

(2) Fundación Instituto de Investigación en Servicios de Salud.

(3) Hospital Virgen del Valle, Toledo.

\section{RESUMEN}

Fundamento: Algunas características sociales, médicas o funcionales se asocian a mayor consumo de recursos sanitarios, sin que esto signifique un uso inapropiado de éstos. El objetivo de este trabajo es conocer la influencia del estado de salud y otros factores sobre las estancias hospitalarias y las estancias inapropiadas en la población mayor de 64 años.

Método: Una cohorte representativa de la población mayor de 64 años del Partido Judicial de Toledo $(n=3.214)$ en la que se había valorado el estado de salud, fue seguida durante 18 meses, identificando sus ingresos y estancias hospitalarias, cuya adecuación fue evaluada con el Appropiateness Evaluation Protocol. Se analizaron las asociaciones entre las características socio-demográficas, de estado de salud y morbilidad de la cohorte con las tasas de frecuentación y hospitalización y con la proporción de estancias e ingresos inadecuados.

Resultados: Durante los 18 meses de seguimiento fueron hospitalizados 410 individuos $(12,8 \%)$, que generaron 546 ingresos (tasa de frecuentación $=17,0$ ingresos/100 habitantes) y 7.015 días de estancia (tasa de hospitalización $=218,3$ estancias/100 habitantes). El 18,9\% de los ingresos y el $49,9 \%$ de las estancias fueron evaluadas como inadecuados. La hospitalización se asoció al peor estado de salud, institucionalización, género masculino, determinadas patologías y a la utilización previa de servicios sanitarios. No se hallaron asociaciones entre características de los pacientes y proporción de estancias inadecuadas. El 97,5\% de las estancias innecesarias fue atribuido a problemas de programación hospitalaria y estilo de practica de los médicos.

Conclusiones: Los factores socio-demográficos, de morbilidad, estado de salud y utilización previa de servicios se muestran como buenos predictores de hospitalización en las personas mayores, pero no se relacionan con el uso inadecuado de la hospitalización.

Palabras Clave: Utilización hospitalaria. Utilización inadecuada. Estado de salud. Ancianos.

Correspondencia:

David Oterino de la Fuente

El Puerto 46

33457 Sta. M. ${ }^{a}$ del Mar - Asturias

Correo electrónico: doterino@eresmas.net

\section{ABSTRACT}

Factors Associated with Hospitalization Use and Appropriateness for Patients Over 64 Years of Age

Background: Some social, medical or functional aspects are associated with a greater use of health care resources, although this does not necessarily imply an inappropriate usage of the same. The aim of this research is that of ascertaining the influence of health condition on hospital stays and inappropriate stays among the population over 64 years of age.

Methods: A cohort representative of the population over age 64 in the Judicial District of Toledo $(n=3214)$ whose health condition had been previously evaluated was studied over an eighteen-month period identifying the income and length of stays at the public hospitals, the appropriateness of which was evaluated by the Appropriateness Evaluation Protocol. Associations analyzed were those of the cohort socio-demographic characteristics, health condition-related variables and morbidity with frequency and hospitalization rates and with inappropriate stays and admissions.

Results: During the 18 months of study 410 individuals were hospitalized, who generated 546 admissions (frequency rate $=17.0$ admissions $/ 100$ rooms) and 7015 stay days (hospitalization rate $=218.3$ stays $/ 100$ rooms). $18.9 \%$ of the admissions and $49.9 \%$ of the stays were evaluated as inappropriate. Hospitalization was associated with a worse health condition, institutionalization, male sex, certain pathologies and previous health services usage. Patients characteristics were not associated with the percentage of inappropriate stays. $97.5 \%$ of inappropriate stays were attributed to hospital scheduling problems and physicians' practices.

Conclusions: Socio-demographic factors, morbidity, health condition and previous health service usage are shown as good hospitalization predictors for senior citizens, but these factors are not related to inappropriate hospitalization usage.

Keywords: Hospital utilization. Inappropiate utilization. Health status. Old people. 


\section{INTRODUCCIÓN}

Las tasas de ingresos y de estancias hospitalarias se incrementan con la edad y de forma más acusada en los individuos mayores de 65 años. Algunas características sociales (vivir solo o en instituciones residenciales), clínicas (enfermedades crónicas), mentales (deterioro cognitivo) y funcionales (pérdida de autonomía para las actividades de la vida diaria) se asocian a un alto consumo de servicios sanitarios, con múltiples ingresos en hospitales de agudos, necesidad de cuidados al alta y problemas para la reinserción en el entorno habitual ${ }^{1-5}$. Esta mayor utilización de servicios sanitarios por los ancianos tiene un fuerte impacto sobre la estructura sanitaria, ya que un $44 \%$ del presupuesto sanitario es consumido por los mayores de 65 años que, además, ocupan el $47 \%$ de las camas de agudos y tienen una estancia media un 32\% superior a la estancia media general ${ }^{6}$.

Aunque algunos estudios han descrito una mayor proporción de uso inadecuado de la hospitalización por la población de más edad ${ }^{7}$, otros trabajos no han relacionado esta variable con una mayor proporción de estancias inadecuadas ${ }^{8}$ e incluso se ha relatado una mayor justificación de los ingresos hospitalarios ${ }^{9,10}$, de las urgen$\operatorname{cias}^{11,12}$ y de la asistencia domiciliaria ${ }^{1}$ en los sujetos más ancianos. Y es que el mayor uso de servicios no puede ser interpretado directamente como uso inadecuado, ya que podría estar justificado por las mayores necesidades de atención de salud derivadas de los problemas inherentes a la edad, como el deterioro mental, alteraciones de la visión, audición y lenguaje, y la mayor prevalencia de enfermedades crónicas y degenerativas ${ }^{13}$. El objetivo de este estudio es conocer la influencia del estado de salud, la comorbilidad y el uso previo de servicios sanitarios en personas de 65 años y más años, sobre el consumo de servicios hospitalarios y el uso inadecuado de la hospitalización.

\section{MATERIAL Y MÉTODO}

Diseño. Cohorte prospectiva, integrada por una muestra representativa de la población mayor de 65 años del Partido Judicial de Toledo (PJT), que fue seguida durante 18 meses (entre el $1 / 10 / 94$ y el $31 / 3 / 96$ ) para identificar los ingresos, estancias hospitalarias y estancias hospitalarias inadecuadas en los hospitales públicos del área sanitaria.

Lugar del estudio. El PJT agrupa a 23 municipios (salvo el de Toledo ninguno supera los 5.000 habitantes), con una población total de 101.900 habitantes, de los cuales $14.862(14,6 \%)$ tenían 65 o más años según el Censo de 1991. El PJT está incluido en el Área Sanitaria de Toledo que cuenta con 2 hospitales públicos: el Hospital Provincial (perteneciente a la Diputación Provincial) y el Complejo Hospitalario de Toledo (INSALUD).

Muestra. La muestra, proveniente de un estudio poblacional para estimar la prevalencia de demencia senil en individuos mayores de 65 años (institucionalizados o no, pero excluyendo los ingresados en hospitales psiquiátricos) del PJT, se obtuvo por medio de un muestreo polietápico estratificado según el índice de ruralidad de los municipios. El tamaño muestral $(n=3.937)$ se estimó para una prevalencia de las demencias menos frecuentes del $0,75 \%$, una precisión del $1 \%$ y un nivel de confianza del 95\%. En personas con déficit cognitivo o con dificultades en el lenguaje la información se obtuvo o se completó a través del cuidador principal. Las características de la muestra y las pérdidas han sido ampliamente descritas en otro trabajo previamente publicado ${ }^{14}$.

Recogida de datos. Los datos sobre las características del paciente, estado de salud y patología autopercibida fueron recogidos por psicólogos y médicos residentes de geriatría, previamente entrenados, mediante entrevista personal con cuestionarios estructurados. Posteriormente se identificaron, a 
través de las bases de datos de los servicios de admisión, las personas de la muestra hospitalizadas durante el seguimiento. No se incluyeron los ingresos en hospitales psiquiátricos, en el Hospital Nacional de Parapléjicos ni en la Unidad de Media Estancia del Hospital del Valle. Para evaluar la adecuación de la hospitalización 8 revisores médicos —entrenados en el uso del Appropiateness Evaluation Protocol (AEP) y que previamente habían alcanzado un Índice de acuerdo observado superior al 90\% (estadísticos Kappa por parejas siempre mayor que 0,85 ) _ revisaron retrospectivamente las historias clínicas de los pacientes que ingresaron y evaluaron la adecuación del ingreso hospitalario y de cada una de las estancias realizadas, salvo - conforme al protocolo $\mathrm{AEP}$ - la correspondiente al día de alta.

Medidas de resultados. Tasa de frecuentación hospitalaria (ingresos de personas de la cohorte en el período de estudio * 100 / población de la muestra); tasa de hospitalización (estancias de personas de la cohorte en el período de estudio * 100 / población de la muestra); proporción de ingresos inadecuados y proporción de estancias inadecuadas, valoradas en ambos casos mediante el Appropriateness Evaluation Protocol (AEP).

Variables e instrumentos. Las variables, definiciones y la mayor parte de los instrumentos utilizados para la medición del estado de salud han sido descritos previamente ${ }^{14}$, por lo que sólo se resumen estos aspectos. Características personales: Edad, sexo, estado civil (casados, sin pareja), domicilio (propio, vive con familiares o cuidadores, residencia de ancianos), nivel de estudios (analfabetos, leer y escribir/sin estudios y estudios primarios, secundarios o superiores), recursos sociales medidos por el Older Americans Resources and Services (OARS, excelentes, buenos, incapacidad social). Patología autorreferida: diabetes, osteoartrosis, hipertensión arterial, patología cardiaca, respiratoria, cataratas, osteoporosis y prostatismo. Estado de Salud: au- topercepción de salud, valorada mediante la pregunta: ¿Durante la semana pasada, cómo se ha encontrado usted de salud? (muy bien/bien, mal/muy mal); autopercepción de dolor, valorado mediante la pregunta: ¿durante la semana pasada ha tenido usted dolor físico? (no he tenido/leve: sin limitación en las actividades básicas vida diaria (AbVD), moderado/intenso: limitación en las $\mathrm{AbVD/requirió} \mathrm{reposo} \mathrm{en} \mathrm{cama);}$ caídas en el último año (ninguna, una o más); estado visual, (sin déficit, déficit leve: dificultad para leer o coser, déficit moderado-severo: dificultad para las actividades de la vida diaria); auditivo (sin déficit, déficit leve: dificultad para conversar en grupo o ambiente ruidoso; déficit moderado-severo: dificultad para conversar con una persona o imposibilidad de conversar); incontinencia urinaria (sí, no); estado cognitivo medido por el Mini-Mental State Examination (MMSE, deterioro cognitivo sí/no a partir de una puntuación de 17/18 para los analfabetos, 20/21 para personas sin estudios y 23/24 para personas con estudios primarios o superiores); AbVD medidas por el Índice de Katz (independientes los individuos que realizaban todas las actividades, dependencia moderada no realizaban 1 actividad, y dependencia severa si no realizaban 2 o más actividades); actividades instrumentales de la vida diaria (AiVD) medidas por el Índice de Lawton (independientes puntuación 8, dependencia moderada entre 4 y 7 , dependencia severa por debajo de 4); depresión medida mediante la Geriatric Depression Scale (GDS: depresión puntuación superior a cinco); calidad de vida valorada mediante el Quality Life Index (QLI: puntuación entre 7 y 10 buena calidad de vida, por debajo de 7 calidad de vida deteriorada); grado de movilidad, valorado según la forma en que podían caminar: sin ayudas, con uno o dos bastones, andador, ayudas de personas o imposibilidad de deambular (siempre que coexistían dos niveles funcionales se aceptaba el peor); se consideró movilidad plena si caminaban solos o con ayuda de un bastón y movilidad restringida el resto de las categorías. Uso previo de servicios sanitarios: 
Ingresos hospitalarios en el año previo a la entrevista (ninguno, uno o más), estancias hospitalarias, y visitas al médico en el mes previo a la entrevista (ninguna, una o más).

Otros instrumentos. Otros instrumentos utilizados y no descritos previamente fueron:

Appropiateness Evaluation Protocol $(\boldsymbol{A E P})^{15-17}$ : Consiste en dos conjuntos de criterios, objetivos e independientes del diagnóstico, destinados a la identificación de admisiones y estancias inapropiadas en pacientes adultos no psiquiátricos. El primer conjunto consta de 16 criterios diseñados para juzgar la necesidad de la admisión hospitalaria y el segundo conjunto, de 27 criterios, se emplea para determinar la necesidad de cada una de las estancias siguientes al día del ingreso. El cumplimiento de uno solo de estos criterios es suficiente para considerar apropiada la admisión o la estancia. El juicio acerca de la necesidad de la admisión o de la estancia se basa en la información disponible en la historia clínica El AEP incluye también una lista de posibles motivos (14 para el ingreso y 22 para la estancia) relacionados con el entorno económico-social y familiar del paciente, la organización del hospital y los servicios, el estilo de practica de los médicos y la infraestructura intra y extrahospitalaria que permite categorizar las causas del uso inapropiado.

Índice de Lawton y Brody ${ }^{18}$ : mide las actividades instrumentales de la vida diaria y es útil para detectar los primeros grados de deterioro. Evalúa ocho actividades: usar el teléfono, ir de compras, preparar la comida, cuidar la casa, lavar la ropa, utilizar transporte, responsabilidad sobre la medicación y capacidad de utilizar dinero. Se puntúa si el individuo realiza la tarea, no si declara que puede hacerla. Puntuación máxima 8 puntos (realiza todas las tareas). El índice de Lawton ha servido de modelo a escalas posteriores y continúa siendo una de las escalas más utilizadas, ya que incluye un rango de actividades más complejas que las que se necesitan para el autocuidado personal, como las que permiten a la persona adaptarse a su entorno y mantener su independencia en la comunidad. Esta escala da mucha importancia a las tareas domésticas, por lo que en nuestro entorno los hombres suelen obtener menor puntuación; no obstante, evalúa la capacidad del individuo para realizar una tarea en una circunstancia determinada, como puede ser la viudedad. La disponibilidad de electrodomésticos y otros utensilios pueden influir también en la puntuación.

Análisis. En primer lugar se analizaron las tasas de hospitalización y de frecuentación en función de las características sociodemográficas, de estado de salud y de morbilidad de los pacientes, valorándose la significación estadística de las diferencias en función del no solapamiento de los intervalos de confianza del $95 \%$ de estas tasas. A continuación se realizaron sendos análisis de regresión lineal múltiple para valorar el efecto de los diversos factores sobre la tasa de hospitalización y la de frecuentación. Se utilizaron modelos de inclusión por pasos (stepwise) con probabilidad de entrada de 0,05 y probabilidad de salida de 0,10 y con incorporación jerárquica de las variables en tres bloques (de estado de salud, de morbilidad y de utilización previa de servicios) que no permitía la exclusión de las variables significativas de los bloques iniciales, aunque los bloques posteriores modificaran su efecto. A continuación se repitió el análisis bivariable entre factores de los pacientes pero usando como variable dependiente la proporción de estancias inadecuadas; no se realizaron análisis multivariantes por la ausencia de asociaciones en el bivariable. Todos los cálculos se realizaron con los programas estadísticos SPSS $®$ y STATA $®$.

\section{RESULTADOS}

Las características personales y el estado de salud de los 3.214 individuos de la muestra han sido previamente descritos ${ }^{14}$. La edad media fue de 74,1 años, existía un pre- 
dominio de mujeres $(56,3 \%)$, un $3,2 \%$ residían en residencias de ancianos, el $66,8 \%$ solo sabían leer y escribir, el 76,5\% eran independientes para realizar las AbVD, y el 65,3 independientes para las AiVD, el $83,6 \%$ referían tener un estado de salud bueno o muy bueno y el 85,9 tenían una buena calidad de vida según el QLI.

Durante los 18 meses de seguimiento de la cohorte fueron hospitalizados en camas de agudos 410 individuos (12,8\%), que generaron 546 ingresos (tasa de frecuentación=17,0 ingresos/100 habitantes) y 7.015 días de estancia (tasa de hospitalización $=218,3$ estancias/100 habitantes). El $75,3 \%$ de los individuos (309) ingresaron una sola ocasión, el 17,8\% (73) dos veces, y el $6,8 \%$ (28) fueron hospitalizados tres o más ocasiones.

La tasa de hospitalización (tabla 1) fue significativamente superior en los individuos mayores de 75 años respecto al grupo de 65-69 años, en los institucionalizados frente a los que vivían en su domicilio, en los incapacitados socialmente frente a los que tenían recursos sociales excelentes, en los que presentaban dependencia severa para las AbVD y AiVD respecto a los independientes, en los que tenían déficit moderado/severo de visión respecto a los que no tenían ningún déficit, y los que tenían deterioro cognitivo, trastornos depresivos, mala calidad de vida, mala o muy mala percepción de salud, dolor moderado/intenso, que habían realizado alguna visita al médico o habían estado en el hospital en el ultimo mes y los que habían sido hospitalizados al menos una vez en el ultimo año. Respecto a la morbilidad (tabla 2), la tasa de hospitalización fue mas elevada en los pacientes con más de tres patologías y los que presentaban afecciones cardiacas o respiratorias. La tasa de frecuentación se comportó de forma muy similar a la de hospitalización, salvo que fueron significativas las diferencias entre hombres y mujeres, y la presencia de patología prostática o cataratas se asoció a una mayor tasa de ingresos.
En el análisis de regresión múltiple (tabla 3) la calidad de vida deteriorada, el vivir en residencias de ancianos, los hombres, la dependencia severa para las actividades básicas de la vida diaria, la autopercepción de dolor incapacitante y la presencia de patología respiratoria o cardiaca se asociaron a la realización de un mayor número de estancias durante el seguimiento. Al incorporar en un bloque posterior las variables de utilización previa de servicios hospitalarios en el mes y en el año anteriores a la entrevista inicial, el sexo, la dependencia y la autopercepción de dolor perdieron su significación estadística. El modelo final explicó un 7,3\% de la varianza en días de hospitalización. El modelo para los ingresos hospitalarios (datos no presentados) incorporó idénticas variables salvo la autopercepción de dolor que fue sustituida por la autopercepción de salud mala o muy mala.

Se obtuvieron las historias de 367 pacientes (6220 estancias; $95,9 \%$ del total) de los 410 que ingresaron en el período de estudio; 26 pacientes (26 estancias) se excluyeron por tratarse de ingresos de un solo día y en 17 casos (265 estancias) no se halló la historia clínica. El 49,9\% $\left(\mathrm{IC}_{95 \%}: 49,2 ; 50,6\right)$ de las estancias revisadas fueron evaluadas como inadecuadas. Las variables referidas al estado de salud de los pacientes, comorbilidad o uso previo de servicios no se asociaron con la proporción de estancias inapropiadas. Respecto a los motivos, el 97,7\% de las estancias inadecuadas se atribuyeron al estilo de práctica de los médicos, las pautas de tratamiento y diagnóstico, la organización interna de los servicios o del hospital y solamente el $2,3 \%$ se atribuyeron a problemas en el entorno familiar o social del paciente $o$ a la falta de estructuras alternativas al hospital (tabla 4). De los 529 ingresos revisados $100(18,9 \%)$ se consideraron inapropiados; en un $48 \%$ se trataba de ingresos para realizar pruebas diagnosticas que podrían hacerse de forma ambulatoria y en un $33 \%$ adicional de ingresos para cirugía electiva con estancia preoperatoria superior a las 24 horas (tabla 5). 
Tabla 1

Tasas de hospitalización y frecuentación

según características personales y de estado de salud

\begin{tabular}{|c|c|c|c|c|c|c|c|c|}
\hline \multirow[b]{3}{*}{ Edad $\dagger$} & \multirow[b]{3}{*}{$65-69$} & \multirow{3}{*}{$\begin{array}{l}n \\
966\end{array}$} & \multicolumn{3}{|c|}{ Hospitalización } & \multicolumn{3}{|c|}{ Frecuentación } \\
\hline & & & \multirow{2}{*}{$\begin{array}{l}\text { tasa } \\
138,0\end{array}$} & \multicolumn{2}{|c|}{ IC $95 \%$} & \multirow{2}{*}{$\begin{array}{l}\text { tasa } \\
11,9\end{array}$} & \multicolumn{2}{|c|}{ IC95\% } \\
\hline & & & & 101,2 & 174,1 & & 9,3 & 14,1 \\
\hline & $70-74$ & 892 & 201,7 & 151,2 & 252,2 & 16,3 & 12,9 & 19,6 \\
\hline & $75-79$ & 616 & 271,7 & 196,1 & 347,0 & 18,5 & 14,3 & 22,7 \\
\hline & 80 o más & 732 & 289,2 & 222,7 & 355,7 & 22,4 & 18,4 & 26,4 \\
\hline \multirow[t]{2}{*}{ Sexo * } & Hombre & 1.404 & 253,6 & 208,2 & 298,9 & 20,2 & 17,3 & 23,1 \\
\hline & Mujer & 1.810 & 190,9 & 156,7 & 225,2 & 14,5 & 12,4 & 16,6 \\
\hline \multirow[t]{2}{*}{ Estado Civil } & Casado & 1.997 & 221,2 & 185,8 & 256,7 & 17,6 & 15,3 & 19,9 \\
\hline & Sin pareja & 1.200 & 216,0 & 171,0 & 260,9 & 16,0 & 13,3 & 18,1 \\
\hline \multirow[t]{3}{*}{ Nivel Estudios } & Analfabeto & 611 & 243,1 & 178,5 & 307,5 & 18,2 & 14,2 & 22,1 \\
\hline & Leer/Escribir & 2.133 & 220,9 & 185,5 & 256,4 & 17,0 & 14,8 & 19,2 \\
\hline & Primarios/Superior & 450 & 166,0 & 112,4 & 219,6 & 14,9 & 10,6 & 19,1 \\
\hline \multirow[t]{3}{*}{ Domicilio $\dagger$} & Propio & 2.568 & 194,0 & 164,8 & 223,2 & 15,4 & 13,5 & 17,2 \\
\hline & Cuidador & 497 & 263,8 & 192,4 & 335,2 & 19,9 & 15,1 & 24,7 \\
\hline & Residencia & 98 & 579,6 & 271,3 & 887,9 & 41,8 & 26,3 & 57,3 \\
\hline \multirow{3}{*}{$\begin{array}{l}\text { Recursos Sociales } \\
\text { (OARS) } \dagger \ddagger\end{array}$} & Excelentes & 1.426 & 184,8 & 147,2 & 222,5 & 14,5 & 12,0 & 17,0 \\
\hline & Buenos & 1.283 & 210,4 & 167,4 & 253,5 & 17,1 & 14,5 & 19,8 \\
\hline & Incapacidad social & 450 & 325,8 & 232,9 & 418,6 & 23,1 & 17,8 & 28,4 \\
\hline \multirow[t]{3}{*}{ Índice de Katz $† *$} & Independiente & 2.595 & 172,5 & 144,5 & 200,4 & 13,9 & 12,2 & 15,8 \\
\hline & Dependencia leve & 376 & 290,8 & 206,9 & 374,8 & 21,3 & 16,4 & 26,3 \\
\hline & Dependencia severa & 228 & 478,8 & 341,8 & 615,9 & 35,1 & 27,0 & 43,3 \\
\hline \multirow[t]{3}{*}{ Índice Lawton $\dagger$} & Independiente & 973 & 128,6 & 89,0 & 168,1 & 10,7 & 8,2 & 13,2 \\
\hline & Dependencia leve & 1.424 & 215,0 & 175,0 & 255,1 & 16,6 & 14,0 & 19,1 \\
\hline & Dependencia severa & 572 & 374,8 & 288,0 & 461,6 & 28,0 & 22,7 & 33,2 \\
\hline \multirow[t]{2}{*}{ Movilidad $\uparrow *$} & Plena & 2.981 & 202,8 & 175,3 & 230,3 & 15,9 & 14,2 & 17,7 \\
\hline & Restringida & 223 & 434,1 & 281,5 & 586,7 & 31,4 & 22,7 & 40,1 \\
\hline \multirow{2}{*}{$\begin{array}{l}\text { Deterioro Cognitivo } \\
\text { (MMSE) } \dagger+\end{array}$} & No & 2.329 & 184,6 & 154,5 & 214,7 & 14,9 & 13,0 & 16,9 \\
\hline & Sí & 864 & 307,4 & 244,9 & 369,9 & 22,3 & 18,6 & 26,0 \\
\hline \multirow[t]{2}{*}{ Depresión $† \ddagger$} & No & 2.400 & 173,9 & 146,0 & 201,9 & 14,0 & 12,2 & 15,8 \\
\hline & Sí & 559 & 327,9 & 241,9 & 413,9 & 23,9 & 18,8 & 29,1 \\
\hline \multirow{2}{*}{$\begin{array}{l}\text { Calidad vida } \dagger \dagger \\
(\mathrm{QLI})\end{array}$} & Buena & 2.713 & 174,0 & 147,6 & 200,4 & 14,2 & 12,4 & 15,9 \\
\hline & Mala & 446 & 460,1 & 348,1 & 572,1 & 32,5 & 26,7 & 38,7 \\
\hline \multirow{2}{*}{$\begin{array}{l}\text { Autopercepción de } \\
\text { Salud } \dagger \ddagger\end{array}$} & Muy Bien/Bien & 2.652 & 190,0 & 161,5 & 218,6 & 15,0 & 13,2 & 16,9 \\
\hline & Mal/Muy Mal & 521 & 346,6 & 260,2 & 433,1 & 28,9 & 20,6 & 31,2 \\
\hline \multirow{2}{*}{$\begin{array}{l}\text { Autopercepción de } \\
\text { Dolor } \dagger+\end{array}$} & No & 2.861 & 191,5 & 164,4 & 218,7 & 15,5 & 13,7 & 17,3 \\
\hline & Sí & 302 & 430,1 & 295,8 & 564,4 & 28,8 & 21,7 & 36,0 \\
\hline \multirow[t]{3}{*}{ Visión †t } & Sin déficit & 2.532 & 194,8 & 166,0 & 223,5 & 15,2 & 13,3 & 17,0 \\
\hline & Déficit leve & 486 & 263,2 & 174,3 & 352,0 & 20,8 & 15,8 & 25,7 \\
\hline & Déficit moderado/severo & 176 & 434,1 & 283,0 & 585,1 & 32,4 & 22,9 & 41,8 \\
\hline Audición & Sin déficit & 2.648 & 207,4 & 177,5 & 237,4 & 16,4 & 14,5 & 18,4 \\
\hline & Déficit leve & 344 & 268,0 & 170,8 & 365,3 & 18,3 & 13,4 & 23,2 \\
\hline & Déficit moderado/severo & 209 & 281,8 & 172,0 & 391,6 & 21,5 & 14,1 & 28,9 \\
\hline
\end{tabular}




\section{Tabla 1 (continuación)}

Tasas de hospitalización y frecuentación según características personales y de estado de salud

\begin{tabular}{|c|c|c|c|c|c|c|c|c|}
\hline \multirow{4}{*}{ Caídas } & \multirow{4}{*}{$\begin{array}{l}\text { Ninguna } \\
\text { Una o más }\end{array}$} & \multirow{3}{*}{$\frac{n}{2.619}$} & \multicolumn{3}{|c|}{ Hospitalización } & \multicolumn{3}{|c|}{ Frecuentación } \\
\hline & & & \multirow{2}{*}{$\frac{\text { tasa }}{211,0}$} & \multicolumn{2}{|c|}{ IC $95 \%$} & \multirow{2}{*}{$\frac{\text { tasa }}{16,1}$} & \multicolumn{2}{|c|}{ IC95\% } \\
\hline & & & & 181,0 & 241,1 & & 14,3 & 18,0 \\
\hline & & 565 & 248,5 & 176,4 & 320,6 & 20,7 & 15,9 & 25,5 \\
\hline \multirow[t]{2}{*}{ Incontinencia } & No & 2.679 & 204,2 & 175,2 & 233,2 & 15,9 & 14,1 & 17,8 \\
\hline & Sí & 509 & 280,7 & 198,9 & 362,6 & 21,8 & 16,7 & 26,9 \\
\hline \multirow[t]{2}{*}{ Visita Médico $†$} & Ninguna & 2.246 & 188,0 & 157,1 & 218,8 & 14,2 & 12,4 & 16,1 \\
\hline & Una o más & 929 & 283,8 & 225,2 & 342,5 & 23,0 & 19,2 & 26,9 \\
\hline \multirow{2}{*}{$\begin{array}{l}\text { Ingresos en el } \\
\text { mes previo } \dagger \ddagger\end{array}$} & Ninguno & 3.125 & 196,7 & 170,6 & 222,8 & 15,7 & 14,0 & 17,4 \\
\hline & Uno o más & 59 & $1.264,4$ & 743,4 & $1.785,4$ & 79,7 & 54,6 & 104,7 \\
\hline \multirow{2}{*}{$\begin{array}{l}\text { Ingresos año } \\
\text { anterior }+t\end{array}$} & Ninguno & 2.825 & 164,3 & 140,3 & 188,3 & 13,2 & 11,6 & 14,7 \\
\hline & Uno o más & 359 & 619,5 & 468,0 & 771,0 & 45,1 & 36,1 & 54,1 \\
\hline
\end{tabular}

$\ddagger \mathrm{p}<0,05$ en tasa de hospitalización; $\uparrow \$ \mathrm{p}<0,05$ en tasa de frecuentación.

Valores perdidos: Edad: 8; Estado civil: 17; Nivel Estudios: 20; Domicilio: 51; Recursos sociales: 55; I. Kazt: 17; I. Lawton: 233; Movilidad: 10; Deterioro cognitivo: 21; Depresión: 255; Calidad de vida: 5; A. Salud: 41; Visión: 20; Audición: 13; Caídas: 30; Incontinencia: 26; V médico: 39; Ingresos mes previo 30; Ingresos año previo: 30 .

Tabla 2

Tasas de hospitalización y frecuentación según patología autorreferida

\begin{tabular}{|c|c|c|c|c|c|c|c|c|}
\hline \multirow{4}{*}{ Diabetes } & \multirow{4}{*}{$\begin{array}{l}\text { No } \\
\text { Sí }\end{array}$} & \multirow{3}{*}{$\frac{n}{2.706}$} & \multicolumn{3}{|c|}{ Hospitalización } & \multicolumn{3}{|c|}{ Frecuentación } \\
\hline & & & \multirow{2}{*}{$\begin{array}{l}\text { tasa } \\
202,8\end{array}$} & \multicolumn{2}{|c|}{ IC $95 \%$} & \multirow{2}{*}{$\frac{\text { tasa }}{16,08}$} & \multicolumn{2}{|c|}{ IC95\% } \\
\hline & & & & 173,2 & 231,9 & & 14,20 & 17,91 \\
\hline & & 436 & 286,1 & 199,6 & 372,4 & 21,10 & 16,15 & 26,06 \\
\hline \multirow[t]{2}{*}{ Osteoartrosis } & No & 1.361 & 242,2 & 196,7 & 287,7 & 18,07 & 15,42 & 20,73 \\
\hline & Sí & 1.809 & 196,1 & 161,8 & 230,3 & 16,03 & 13,71 & 18,36 \\
\hline Hipertensión & No & 1.989 & 203,7 & 169,1 & 238,2 & 16,24 & 14,10 & 18,38 \\
\hline Arterial & Sí & 1.139 & 236,4 & 189,4 & 283,5 & 17,56 & 14,59 & 20,53 \\
\hline \multirow[t]{2}{*}{ Patología Cardiaca $\dagger \sharp$} & No & 2.634 & 177,4 & 150,1 & 204,8 & 13,78 & 12,08 & 15,49 \\
\hline & Sí & 580 & 396,0 & 309,5 & 482,6 & 31,55 & 25,99 & 37,11 \\
\hline \multirow[t]{2}{*}{ Patología Respiratoria $\dagger$} & No & 2.717 & 186,7 & 158,7 & 214,7 & 14,54 & 12,80 & 16,28 \\
\hline & Sí & 467 & 377,9 & 286,2 & 469,7 & 30,19 & 24,08 & 36,30 \\
\hline \multirow[t]{2}{*}{ Cataratas } & No & 2.188 & 192,7 & 162,8 & 222,6 & 15,08 & 13,13 & 17,04 \\
\hline & Sí & 964 & 262,7 & 206,0 & 319,3 & 20,95 & 17,38 & 24,53 \\
\hline \multirow[t]{2}{*}{ Osteoporosis } & No & 2.543 & 213,1 & 182,3 & 243,9 & 16,63 & 14,69 & 18,58 \\
\hline & Sí & 424 & 254,0 & 171,6 & 336,4 & 19,81 & 14,66 & 24,97 \\
\hline \multirow[t]{2}{*}{ Prostatismo $\ddagger$} & No & 2.784 & 205,4 & 176,1 & 234,7 & 16,02 & 14,24 & 17,80 \\
\hline & Sí & 409 & 310,7 & 224,7 & 396,8 & 24,21 & 17,99 & 30,42 \\
\hline \multirow[t]{2}{*}{ Número Patologías $\dagger$ † } & $0-2$ & 1.483 & 163,6 & 127,3 & 199,9 & 12,34 & 10,25 & 14,43 \\
\hline & 3 ó más & 1.720 & 261,6 & 221,0 & 302,2 & 20,81 & 18,15 & 23,48 \\
\hline
\end{tabular}

$\$ \mathrm{p}<0,05$ en tasa de hospitalización; $\uparrow \$ \mathrm{p}<0,05$ en tasa de frecuentación.

Valores perdidos: Diabetes: 72; Osteoartrosis: 44; Hipertensión: 86; EPOC: 30; Cataratas: 62; Osteoporosis: 247; Prostatismo: 21. 
Tabla 3

Factores asociados a la hospitalización (días de estancia en el período de estudio). Análisis regresión múltiple

\begin{tabular}{|l|c|c|cc|c|c|}
\hline & $\beta$ & $D E(\beta)$ & IC 95\% & $(\beta)$ & $\beta$ est. & $p$ \\
\hline Quality Life Index < 7 puntos & 1,126 & 0,555 & 0,068 & 2,184 & 0,049 & 0,043 \\
Vivir en residencia & 2,577 & 0,884 & 0,893 & 4,262 & 0,056 & 0,004 \\
Sexo hombre & 0,448 & 0,304 & $-0,131$ & 1,028 & 0,028 & 0,140 \\
Índice Kazt (dependencia severa) & 0,414 & 0,288 & $-0,135$ & 0,964 & 0,033 & 0,150 \\
Autopercepción dolor (incapacitante) & 0,706 & 0,559 & $-0,359$ & 1,771 & 0,026 & 0,206 \\
\hline Patología Respiratoria & 1,247 & 0,425 & 0,436 & 2,058 & 0,055 & 0,003 \\
Patología Cardiaca & 1,210 & 0,392 & 0,462 & 1,958 & 0,058 & 0,002 \\
\hline Ingreso mes previo entrevista basal & 7,684 & 1,141 & 5,509 & 9,859 & 0,129 & $<0,001$ \\
Ingreso año previo entrevista basal & 2,910 & 0,494 & 1,968 & 3,852 & 0,115 & $<0,001$ \\
\hline Constante & $-0,447$ & 0,687 & $-1,758$ & 0,864 & & 0,515 \\
\hline
\end{tabular}

$\mathrm{n}=2754 ; \mathrm{R}^{2}=0,073 ;$ Método Stepwise; $\beta$ : coeficiente beta; DE: desviación estándar; IC 95\%: Intervalo de confianza del $95 \%$; $\beta$ est: coeficiente beta estandarizado.

Tabla 4

Motivos de las estancias inapropiadas

\begin{tabular}{|c|c|c|c|}
\hline & & $n$ & $\%$ \\
\hline \multirow{6}{*}{$\begin{array}{l}\text { Problemas programación en pa- } \\
\text { cientes que requieren continuar } \\
\text { ingresados en el hospital } \\
\text { n: } 752(24,2 \%)\end{array}$} & Problemas programación intervención quirúrgica & 63 & 2,03 \\
\hline & Problemas programación pruebas o procedimientos diagnósticos & 164 & 5,28 \\
\hline & Admisión prematura & 49 & 1,58 \\
\hline & No disponibilidad de quirófano & 29 & 0,93 \\
\hline & Procedimientos que no se realizan en fin de semana, vacaciones, etc. & 40 & 1,29 \\
\hline & Retraso resultados pruebas o interconsultas necesarias para evaluar el alta & 407 & 13,09 \\
\hline \multirow{6}{*}{$\begin{array}{l}\text { Demora alta por problemas bajo } \\
\text { la responsabilidad del hospital o } \\
\text { del médico } \\
\text { n: } 2.279(73,5 \%)\end{array}$} & Demora en escribir la orden de alta & 48 & 1,54 \\
\hline & Demora en iniciar la orden de alta & 39 & 1,25 \\
\hline & Pauta medica conservadora & 1.457 & 47,06 \\
\hline & Falta plan documentado de diagnostico $\mathrm{y} / \mathrm{o}$ tratamiento & 345 & 11,10 \\
\hline & Retraso resultados pruebas NO necesarias para evaluar el alta & 375 & 12,06 \\
\hline & Otros & 15 & 0,48 \\
\hline \multirow{4}{*}{$\begin{array}{l}\text { Demora alta por problemas del } \\
\text { entorno del paciente } \\
\text { n: } 41(1,3 \%)\end{array}$} & No hay familiares para cuidar al paciente en el domicilio & 12 & 0,39 \\
\hline & La familia no esta preparada para cuidar al paciente & 1 & 0,03 \\
\hline & El paciente/familia rechazan un alta con cuidados alternativos adecuados & 13 & 0,42 \\
\hline & Otros & 15 & 0,48 \\
\hline \multirow{2}{*}{$\begin{array}{l}\text { Demora alta por falta alternati- } \\
\text { vas hospital } \\
\text { n: } 31(1,0 \%)\end{array}$} & No se dispone de plazas en centros alternativos & 24 & 0,77 \\
\hline & No existen centros alternativos & 7 & 0,23 \\
\hline Total & & 3.103 & 100 \\
\hline
\end{tabular}


Tabla 5

Motivos de ingresos inapropiados

\begin{tabular}{|rlr|}
\hline \multicolumn{1}{|c|}{ Motivos } & $n$ & $\%$ \\
\hline 1. Pruebas diagnósticas y/o tratamiento pueden realizarse ambulatoriamente & 48 & 48 \\
2. El paciente vive muy lejos & 2 & 2 \\
3. La realización de las pruebas ambulatorias tiene mucho retraso & 4 & 4 \\
4. El paciente requiere ingreso en un nivel inferior & 1 & 1 \\
5. El paciente requiere cuidados en un hospital de crónicos & 1 & 1 \\
6. El paciente requiere cuidados de enfermería & 2 & 2 \\
8. Admisión prematura & 33 & 33 \\
9. Tratamiento o pruebas no documentados & 2 & 2 \\
10. Procedimiento quirúrgico podría realizarse de forma ambulatoria & 1 & 1 \\
11. Necesidad de cuidados terminales & 1 & 1 \\
12. Paciente ingresado para custodia & 2 & 2 \\
13. Incumplimiento del tratamiento necesario & 1 & 1 \\
19. Otros & 2 & $\mathbf{1 0 0}$ \\
\hline Total & $\mathbf{1 0 0}$ \\
\hline
\end{tabular}

\section{DISCUSIÓN}

Los resultados de este estudio muestran que la tasa de hospitalización en la población de 65 y más años se asocia con un estado de salud bajo, a la institucionalización, al sexo masculino, a determinadas patologías y a la utilización previa de servicios sanitarios. Adicionalmente, muestran una proporción de estancias inadecuadas en el límite alto de las encontradas en otros estudios realizados sobre población general o en ancianos ${ }^{12,19-22}$ que no se asocia al estado de salud, comorbilidad, ni uso previo de servicios sanitarios.

La tasa de hospitalización es inferior a la obtenida en la Encuesta de Morbilidad Hospitalaria ${ }^{23}$ y a la de otro estudio realizado en Cataluña ${ }^{24}$, que incluyen ingresos en hospitales psiquiátricos, privados y otros no considerados en este estudio, al margen de la menor utilización de servicios en un territorio mayoritariamente rural. Adicionalmente, la tasa de hospitalización podría estar infravalorada por perdidas en la identificación de personas ingresadas, ingresos en hospitales de otras Áreas Sanitarias y por la el mante- nimiento en el denominador de la tasa de fallecidos y otras perdidas de la cohorte.

Los factores asociados a la mayor utilización hospitalaria son consistentes con la literatura previa. Los hombres — si se excluye la atención maternal - realizan más ingresos y estancias hospitalarias en todos los grupos de edad $^{25-27}$. La edad se asocia a un mayor riesgo de hospitalización ${ }^{28}$, aunque pierde importancia cuando se ajusta por el estado de sa$\operatorname{lud}^{25,29-32}$. El estado de salud aparece fuertemente asociado al consumo de servicios hospitalarios $^{25,26,28,29}$ sea cual sea el indicador que se utilice (OARS, Katz, Lawton, GDS, QLI, autopercepción de salud y dolor, etc.); la elevada correlación entre estos indicadores hace que muchos de ellos sean desplazados de los modelos multivariantes, aunque los indicadores más multidimensionales como el $\mathrm{QLI}^{33,34}$, el Katz ${ }^{35-37}$ o la autopercepción de salud/dolor parecen mantener una mayor capacidad predictiva sobre la hospitalización. Cabe destacar que «dependencia para las ABVD» desplaza la «dependencia para las AIVD» medidas por el índice de Lawton, probablemente porque el índice de Katz es más específico para detectar la discapacidad moderada/severa que el índice de Lawton, 
aunque éste sería más sensible para detectar pequeñas pérdidas en la capacidad funcional (usar el teléfono, hacer la compra,...) en las personas previamente independientes que viven en la comunidad ${ }^{35}$. La institucionalización también se asocia a mayor consumo de servicios hospitalarios, asociación que se mantiene incluso tras controlar por las variables «estado de salud», «presencia de patologías» y «utilización previa». Se ha señalado que las personas que viven en residencias tienen una prevalencia de deterioro funcional y cognitivo más elevada que las que viven en la comunidad ${ }^{38}$ y en este estudio las personas institucionalizadas tenían peor puntuación en todos los indicadores de estado de salud. La patología cardiaca y la enfermedad pulmonar obstructiva crónica son, junto a los accidentes cerebrovasculares, los diagnósticos más frecuentes en los ancianos hospitalizados y los que originan una mayor mortalidad, incapacidad y también un mayor consumo de los cuidados post-hospitalarios, habiéndose reportado para Estados Unidos, que dicho consumo llega al $60 \%{ }^{39}$. Las enfermedades cardiacas son las que se han relacionado más fuertemente con la probabilidad que un individuo tiene de ser hospitalizado ${ }^{28,31,40}$. Finalmente, la utilización previa de servicios hospitalarios es un predictor conocido de nuevas utilizaciones posteriores, asociándose también a mayor mortalidad, mayor deterioro funcional y a la estancia en residencias de an$\operatorname{cianos}^{26,28,31}$.

Respecto a la utilización inadecuada, la no asociación con ninguna de las características de los sujetos sugiere que esta variable depende, sobre todo, de los estilos de práctica de los clínicos y la organización hospitalaria. Esta explicación es consistente con la distribución de los motivos de uso inadecuado (tablas 4 y 5) y orienta hacia la necesidad de mejorar los procesos de alta y la programación de pruebas e intervenciones para agilizar su realización, evitando la prolongación de la estancia o el propio ingreso. No obstante, cuando un paciente que podría ser atendido en su domicilio o en un centro socio-sanitario es mantenido en el hospital sin que se le dé el alta, la responsabilidad sobre la inadecuación es achacable al médico o al hospital, ya que no existen problemas familiares ni déficit en la red alternativa, puesto que no se ha requerido su colaboración. Una hipótesis alternativa que podría explicar parcialmente los resultados hallados sería que la proporción de estancias inapropiadas atribuidas al medico o al hospital esconda una proporción indeterminada de estancias inapropiadas atribuibles a otras causas, que sólo serán identificadas cuando estos recursos alternativos sean requeridos.

Entre las limitaciones del estudio hay que señalar, en primer lugar, la no consideración de las pérdidas de la cohorte por mortalidad (o cambio de domicilio), aspecto que probablemente produce una atenuación del impacto de muchos de los factores analizados sobre la hospitalización, ya que las personas fallecidas tenían previsiblemente peores indicadores ${ }^{41,42}$ pero al haber fallecido no pueden hacer uso de los servicios hospitalarios. Otras limitaciones a tener en cuenta es el elevado volumen de pruebas estadísticas realizadas sin correcciones para pruebas múltiples, lo que puede haber favorecido la presencia de asociaciones espúreas y el no cumplimiento de algunos supuestos de los modelos de regresión lineal. En cuanto al AEP, se trata de un instrumento caracterizado por su alta fiabilidad y adecuada validez para la identificación del uso hospitalario inapropiado ${ }^{17}$ pero que, al igual que otros instrumentos destinados a evaluar la necesidad de hospitalización, se aplican bajo determinadas asunciones y presentan algunas limitaciones ampliamente descritas en trabajos específicos ${ }^{17,43}$. Entre ellas hay que citar la necesidad de una cierta calidad en la confección de las historias clínicas, la asunción de que los cuidados prestados son siempre apropiados desde el punto de vista clínico, de que sólo los aspectos clínicos y algunos factores sociales que afectan a la evolución clínica justifican el ingreso hospitalario, o de que todos los niveles de cuidados se hallan potencialmente disponi- 
bles. Finalmente, en cuanto a las limitaciones de la generalización de los resultados, debe tenerse en cuenta que la hospitalización inadecuada está relacionada con la oferta de camas geriátricas y generales, la organización de los hospitales, la duración de la estancia, y con otros factores, por lo que su proporción varía notoriamente de un lugar a otro.

Como conclusión, hay que resaltar que diversos factores socio-demográficos, de morbilidad, estado de salud y utilización previa de servicios, se muestran como buenos predictores de hospitalización en las personas mayores, pero no de adecuación de las estancias. El primer aspecto permite valorar el riesgo de hospitalización y la necesidad de recursos en función de diversos indicadores, resaltando el valor de los relacionados con el estado de salud; el segundo refuerza la hipótesis de que el uso inadecuado de la hospitalización no se relaciona con las características de los pacientes, sino con la organización del hospital, la programación de los procesos y los estilos de práctica.

\section{BIBLIOGRAFÍA}

1. González Montalvo JI, Pérez del Molino J, Rodríguez Mañas L, Salgado Alba A, Guillén Llera F. Geriatría y asistencia geriátrica: para quién y cómo (I). Med Clin (Barc) 1991;96:183-8.

2. Sager MA, Franke T, Inouye SK, Landefeld CS, Morgan TM, Rudberg MA et al. Functional outcomes of acute medical illness and hospitalization in older persons. Arch Intern Med 1996;156:645-52.

3. Hirsch C, Sommers L, Olsen A, Mullen L, Wino$\operatorname{grad} \mathrm{CH}$. The natural history of functional morbidity in hospitalized older patients. J Am Geriatr Soc 1990; 38: 1296-1303.

4. Freeborn DK, Pope CR, Mullooly JP, McFarland $\mathrm{BH}$. Consistently high users of medical care among the elderly. Med Care 1990; 28: 527-40.

5. Gené J, Contel J C, Tintoré I, Villafáfila R, Schorlemmer C. Salud y utilización de servicios en ancianos, según el nivel de convivencia. Gac Sanit 1997; 11: 214-20.
6. INSALUD. Dirección General de Organización y Planificación Sanitaria. Nivel de Salud y utilización de recursos. En: Programa de atención a las personas mayores. Madrid: INSALUD, 1999:35-50.

7. Mozes B, Schiff E, Modan B. Factors affecting inappropriate hospital stay. Qual Assur Health Care 1991;3:211-7.

8. Portella E, Oterino de la Fuente D, Peiro S. Impact de l'agé sur la utilization non necesaire. En: La dependence, defi de l'an 2000. Geneve. ed. JC Rey, C Tilquin; 1994:174-79.

9. Muiño A, González VJ, Rodríguez E, Lázaro C, Fernández E. Asistencia en un servicio de urgencia: justificación de las visitas y adecuación de los ingresos. Rev Clin Esp 1988;182:374-8.

10. González Montalvo JI, Baztán JJ, Rodríguez Mañas L, San Cristóbal E, Gato A, Ballesteros P et al. Ingreso hospitalario urgente en servicios médicos, justificación, factores sociales y sus diferencias en función de la edad. Med Clin (Barc) 1994;103:441-44.

11. Rodríguez F, González J I, Sanz F, Jaramillo E, Banegas JR, Rodríguez L, et al. La urgencia geriátrica frente a la urgencia en edad adulta: análisis retrospectivo de las urgencias médicas de un hospital. Med Clin (Barc) 1989;93: 411-4.

12. Oterino de la Fuente D, Peiró S, Marchan C, Porte1la E. Inappropiate Hospitalisation: reasons and determinants. Eur J Public Health 1996;6:126-132.

13. Zunzunegui MV. Envejecimiento y Salud. En: Informe SESPAS 1998: la Salud Pública y el futuro estado del bienestar. Granada: Escuela Andaluza de Salud Pública-SESPAS; 1998. p. 121-39.

14. Suárez García F, Oterino de la Fuente D, Peiró S, García García F, Librero J, Pérez Martín A, Martín Correa E, Serrano Lira F. Estado de salud de las personas ancianas y hospitalización en servicios geriátricos, médicos y quirúrgicos. Estudio poblacional en Toledo. Rev Esp Salud Pública 2000;74:149-61.

15. Gertman PM, Restuccia JD. The Appropriateness Evaluation Protocol: a technique for assessing unnecessary days of hospital care. Med Care 191;19:855-71.

16. Restuccia JD, Payne S, Lenhart G, Fulton J. Assessing the appropriateness of hospital utilization to improve efficiency and competitive position. Health Care Manage Rev 1987,12:17-27. 
17. Peiró S, Meneu R, Roselló ML, Portella E, Carbonell-Sanchís R, Fernández C et al. Validez del protocolo de evaluación del uso inapropiado de la hospitalización. Med Clin (Barc) 1996;107:124-29.

18. Lawton MP, Brody EM. Assessment of older people: self maintaining and instrumental activities of daily living. Gerontologist 1969;9:179-86.

19. Booth BM, Ludke RL, Wakefield DS, Kern DC, Burmeister LF, Fisher EM, et al. Nonacute days of care within Department of Veterans Medical Centers. Med Care 1991;29 Supl 8:AS51-AS63.

20. Siu AL, Manning WG, Benjamin B. Patients provider and hospital characteristics associated with inappropriate hospitalization. Am J Public Health 1990;80:1253-56.

21. DeCoster C, Roos N, Carrière KC, Peterson S Inappropiate hospital use by patients receiving care for medical conditions: targeting utilization review. Can Med Assoc J 1997;157:889-96.

22. Ochoa P, García-Arilla E. Admisión y estancia inapropiada según el Appropriateness Evaluation Protocol. Estudio de prevalencia de punto en un servicio de geriatría. Med Clin (Barc) 1997;109:195

23. Instituto Nacional de Estadística. Encuesta de Morbilidad Hospitalaria 1996. Madrid 1997

24. Castellá X, Mompart A, Pérez G. La utilización de los hospitales de agudos por los ancianos. Cataluña, 1982-1990. Gac Sanit 1997;11:259-65.

25. Noro AM, Häkkinen UT, Laitinen OJ. Determinants of heath service use and expenditure among the elderly Finnish population. Eur J Pub Health 1999;9:174-80.

26. Wolinsky FD, Johnson RJ. The use of health services by older adults. J Gerontol Soc Sci 1991;46: S345-357

27. McCusker J, Healey E, Bellavance F, Connolly B. Predictors of repeat emergency department visits by elders. Acad Emerg Med 1997;4:581-8.

28. Boult C, Dowd B, McCaffrey D, Boultn L, Hernández R, Krulewitch $\mathrm{H}$. Screening elders for risk of hospital admission. J Am Geriatr Soc 1993;41:811-7.

29. Abizanda P, Luengo C, López-Torres J, Sánchez $\mathrm{P}$, Romero L, Fernández C. Predictores de mortalidad, deterioro funcional e ingreso hospitalario en una muestra de ancianos residentes en la comunidad. Rev Esp Geriatr Gerontol 1998;33:219-5.
30. Tomiak M, Berthelot JM, Mustard CA. A profile of health care utilization of the disabled population in Manitoba. Med Care 1998;36:1383-97.

31. Wolinsky FD, Culler SD, Callahan CM, Johnson RJ. Hospital resource consumption among older adults: a prospective analysis of episodes, lengh of stay, and charges over a seven-year period. J Gerontol 1994; 49: S240-52.

32. Gruenberg L, Tompkins C, Porell F. The health status and utilization patterns of the elderly: implications for setting Medicare payments to HMOs. Adv Health Econ Health Serv Res 1989;10:41-73.

33. Siu AL, Reuben DB, Ouslander JG, Osterweil D Using multidimensional health measures in older persons to identify risk of hospitalization and skilled nursing placement. Qual Life Res $1993 ; 2: 253-61$.

34. Alonso J. La medida de la calidad de vida relacionada con la salud en la investigación y la práctica clínica. Gac Sanit 2000; 14: 163-167.

35. Guralnik JM, Fried LP, Salive ME. Disability as a public health outcome in the aging population. Annu Rev Public Health 1996;17:25-46.

36. Mor V, Wilcox V, Rakowski W, Hiris J. Functional transitions among the elderly: patterns, predictors, and related hospital use. Am J Public Health 1994;84:1274-80.

37. Covinsky KE, Justice AC, Rosenthal GE, Palmer RM, Landefeld CS. Measuring prognosis and case mix in hospitalized elders. The importance of functional status. J Gen Intern Med 1997;12:203-8.

38. IMSERSO. Resultados descriptivos. En Atención a las personas mayores que viven en residencias. Madrid 1998

39. Harrison CR. Prospective payment for medicare posthospital services. Some empirical considerations. Santa Mónica: RAND Corporation; 1986.

40. Blaum CS, Liang J, Liu X. The relation of chronic diseases and health status to the health services utilization of older americans. J Am Geriatr Soc 1994;42:1087-93

41. Guralnik JM, LaCroix AZ, Branch LG, Kasl SV, Wallace RB. Morbidity and disability in older persons in the years prior to death. Am J Public Health 1991;81:443-7.

42. Stearns SC, Kovar MG, Hayes K, Koch GG. Risk indicators for hospitalization during the last year of life. Health Serv Res 1996;31:49-69.

43. Lorenzo S. Métodos de revisión de utilización de recursos: limitaciones. Med Clin (Barc) 1996; 107: $22-25$.

Rev Esp Salud Pública 2001, Vol. 75, N. ${ }^{\circ} 3$ 